4. Cameron NE, Cotter MA, Low PA (1991) Nerve blood flow in early experimental diabetes in rats: relation to conduction deficits. Am J Physiol 261: E1-E8

5. Kohner EM, Hamilton AM, Saunders SJ et al. (1975) The retinal blood flow in diabetes. Diabetologia 11: 27-33

6. Mogensen CE (1971) Glomerular filtration rate and renal plasma flow in short-term and long-term juvenile diabetes mellitus. Scand J Clin Lab Invest 28: 91-100

7. Houben AJHM, Schaper NC, Slaaf DW et al. (1992) Skin blood cell flux in insulin-dependent diabetic subjects in relation to retinopathy or incipient nephropathy. Eur J Clin Invest 22: 67-72

8. Tilton RG, Chang K, Hasan KS et al. (1993) Prevention of diabetic vascular dysfunction by guanidines-inhibition of nitric oxide synthase versus advanced glycation end-product formation. Diabetes 42: 221-232

9. Corbett JA, Tilton RG, Chang K et al. (1992) Aminoguanidine, a novel inhibitor of nitric oxide formation, prevents diabetic vascular dysfunction. Diabetes 41: 552-556

\section{Response from the authors}

Dear Sir,

We are grateful to Drs. Wascher and Toplak for their comments about our work involving photography of sural nerve vessels and fluorescein angiography. We accept their comment that there is a need to make similar observations in nonneuropathic diabetic patients who do not obviously have microvascular complications, but as the procedure involved was significantly invasive, although with no long-term complications, the number of non-neuropathic subjects volunteering to undergo the procedure during the study period was rather limited. This was in contrast to the number of neuropathic patients who were often very willing to volunteer for the procedure. We are now in the process of developing techniques for measuring nerve blood flow including fluorescein angiography through a

Corresponding author: Dr. S. Tesfaye, The Diabetes Center, Walton Hospital, Rice Lane, Liverpool L9 IAE, UK
10. Graier WF, Wascher TC, Lackner L et al. (1993) Exposure to elevated D-glucose concentrations modulates vascular endothelial cell vasodilatory response. Diabetes 42: 1497 1505

11. Williamson JR, Chang K, Frangos M et al. (1993) Hyperglycemic pseudohypoxia and diabetic complications. Diabetes 42: 801-813

12. Cameron NE, Cotter MA, Dines KC et al. (1992) Effect of aminoguanidine on peripheral nerve function and polyol pathway metabolites in streptozotocin-diabetic rats. Diabetologia 35: 946-950

13. Cameron NE, Cotter MA, Maxfield MA (1993) Antioxidant treatment prevents the development of peripheral nerve dysfunction in streptozotocin-diabetic rats. Diabetologia 36: 299-304

14. Cameron NE, Cotter MA (1992) Impaired contraction and relaxation in aorta from streptozotocin-diabetic rats: role of polyol pathway. Diabetologia 35: 1011-1019 much reduced incision as the technique of light-guide spectrophotometry and laser Doppler has been developed and miniaturised.

The other point raised by Drs. Wascher and Toplak was the need to match glycaemic control when comparing indices of blood flow. There was no significant difference in either mean $\mathrm{HbA}_{1}$ or mean ambient blood glucose between both the diabetic groups, nor was there any correlation between the intensity of fluorescence and $\mathrm{HbA}_{1}$, although admittedly the numbers could have been too small for a decent correlation.

We accept there is a great need to investigate the effect of various therapeutic agents on nerve blood flow (gamma linolenic acid, angiotensin converting enzyme inhibitors) and we hope that our miniaturised technique will allow such studies.

Yours sincerely,

S. Tesfaye, J.D. Ward

\title{
Autonomic neuropathy: comparison of two screening procedures
}

Dear Sir,

The EURODIAB IDDM Complications Study Group reported on the frequency of microvascular and acute complications in patients with insulin-dependent diabetes mellitus [1]. We would like to reflect on autonomic test results. Two tests were applied in the above-mentioned study: changes in heart rate (30/15 ratio) and in systolic blood pressure on standing. In $19.3 \%$ of patients the $30 / 15$ ratio was abnormal, while postural hypotension was observed in only $5.9 \%$ of patients. Corresponding data in the Hungarian cohort were $37.1 \%$ and $7.9 \%$, respectively.

Corresponding author: Dr. P. Kempler, I.Dept. of Medicine, Semmelweis University, Korányi S. u. 2/a, H-1083 Budapest, Hungary
The aim of the present study was to compare the efficacy of two different screening procedures: those of the EURODIAB IDDM Complications Study with those of our self-developed protocol.

We examined 206 IDDM patients (mean age: 32.2; range: 14-57 years, mean duration of diabetes: 11.2 ; range: 0 43 years). Heart rate responses induced by deep breathing, Valsalva manoeuvre and standing up (35/15 ratio) and changes in blood pressure induced by standing and sustained handgrip were evaluated [2]. Eighty-three patients ( $40 \%$ ) had normal results in all five tests. Thirty-five patients $(17 \%)$ had one abnormal refiex test and 88 patients $(43 \%)$ had more than one abnormal reflex test. Abnormal heart rate response to deep breathing (in 84 patients) as well as abnormal 30/15 ratio (in 80 patients) were found significantly more often than an 
abnormal Valsalva ratio (in 18 patients, $p<0.001$ at both comparisons, McNemar test). Abnormal handgrip test (in 73 patients) was found significantly more often than an abnormal fall in blood pressure after standing up (in 3 patients, $p<0.001$, McNemar test). Abnormal blood pressure tests were present only if at least one of the heart rate tests was abnormal.

Data were analysed according to two different protocols. By the EURODIAB protocol 38 patients $(18 \%$ ) had false negative results: they had normal $30 / 15$ ratio and normal blood pressure response to standing; however, in 18 patients one, and in 20 patients, more than one of the three other tests were abnormal.

According to our protocol, heart rate response to deep breathing and 30/15 ratio were examined. In this case, only three patients $(1.5 \%)$ showed false-negative results, only the Valsalva ratio being abnormal. Both heart rate responses to deep breathing and standing were abnormal in 45 of our 206 patients. However, 32 of these 45 patients had abnormal results in at least one of the three other tests as well, indicating moderate to severe autonomic involvement. Thus, evaluation of these two tests also seems to provide some possibility for the assessment of severity of autonomic dysfunction.

Both protocols examined the 30/15 ratio. Evaluation of heart rate response to deep breathing instead of blood pressure response to standing seems to be a useful alternative way for screening autonomic function. Both tests equally require $60 \mathrm{~s}$.

Recently, we suggested evaluating heart rate responses to deep breathing and standing as the first step of diagnosis, as part of our rational diagnostic model for the assessment of autonomic function [3]. Indeed, for screening purposes in large epidemiological studies, examination of these two tests heart rate responses to deep breathing and standing - seems to be sufficient.

Yours sincerely,

P. Kempler, Zs. Kerényi, Gy. Tamás

\section{References}

1. EURODIAB IDDM Complications Study Group (1994) Microvascular and acute complications in IDDM patients: the EURODIAB IDDM Complications Study. Diabetologia 37: 278-285

2. Ewing DJ, Clarke BF (1982) Diagnosis and management of diabetic autonomic neuropathy. BMJ 285: 916-918

3. Kempler P, Váradi A, Tamás Gy (1990) Which battery of cardiovascular autonomic function test - suggestion for a rational diagnostic model. Diabetologia 33: 650

\section{Prevalence of maternally inherited diabetes and deafness in diabetic populations in the Netherlands}

\begin{abstract}
Dear Sir,
Previously we described a mutation in the mitochondrial encoded tRNA ${ }^{\text {Leu(UUR) }}$ gene at position 3243 which cosegregated with non-insulin-dependent diabetes mellitus (NIDDM) and an associated deafness [1]. Additional publications have corroborated this finding [2-4]. Furthermore, we found that this mutation, which is also found in patients with mitochondrial myopathy, encephalopathy, lactic acidosis and strokelike episodes (MELAS), is most likely the only mutation responsible for this subtype of diabetes, for which we proposed the name: Maternally Inherited Diabetes and Deafness (MIDD) [4]. Recently the prevalence of this mutation in selected French pedigrees with multiplex NIDDM was reported to be approximately $2 \%$ [5]. Katagiri et al. [2] showed that the prevalence of this mitochondrial tRNA ${ }^{\text {Leu(UUR) }}$ mutation in a Japanese group of subjects selected for maternal inheritance of diabetes is slightly more than $1 \%$. Another study by Kadowaki et al. [3] indicated a prevalence of respectively $6 \%$ and $2 \%$ in Japanese patients with familial IDDM or NIDDM.

In this letter we present the prevalence of the tRNA ${ }^{\mathrm{Leu}(\mathrm{UUR})}$ gene mutation in two NIDDM populations in the Netherlands: one population consisting of 473 randomly selected NIDDM patients from three population-based studies in the Netherlands (The 'Hoorn Study', the 'Zutphen Study' and the 'Rotterdam Study'). All patients in this group were of Caucasian origin and over 40 years of age. Diabetes was diagnosed by oral glucose tolerance test according to World Health Organi-
\end{abstract}

Corresponding author: Dr. J. A. Maassen, Department of Medical Biochemistry, Wassenaarseweg 72, P.O. Box 9503, NL2300 RA Leiden, The Netherlands zation criteria. Diabetic patients with marked insulinopenia were excluded from the present study, i.e. when requiring insulin treatment within one year after diagnosis of diabetes.

The other group comprised 28 pedigrees from the diabetes clinic of the University Hospital, Leiden, selected for the presence of NIDDM and deafness in at least two generations, and with a mode of inheritance agreeing with a maternal one. Total DNA isolation from leucocytes and detection of the 3243 mutation in the mitochondrial DNA was as described earlier [4].

In the population-based group we detected two subjects out of 473 carrying the mutation, A020 and A135 (pedigrees shown in Fig. 1). Upon further investigation of these two pedigrees it appeared that individual A020 (age 27 years) suffers from a growth hormone deficiency. Her brother, mother, ma-
A

$\mathrm{A} 020$

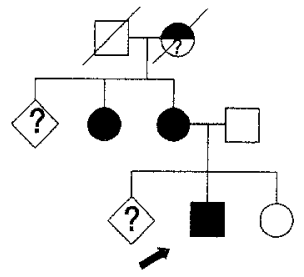

B

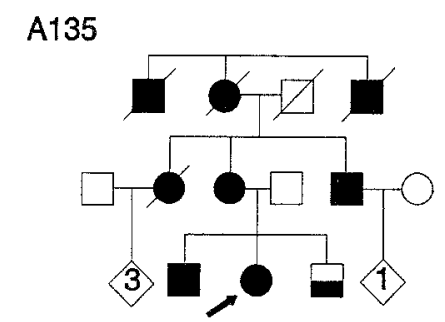

Fig. 1. Relevant parts of pedigrees. A. Patient A020. B. patient A135. Probands are indicated with an arrow. $\odot$ NIDDM: $\odot$ deafness; NIDDM and deafness; ? unknown 